\title{
Impairment of cardiomyogenesis in embryonic stem cells lacking scaffold protein JSAP
}

\author{
T. Sato, K. Hidaka, A. Iwanaga, M. Ito, M. Asano, Y. Nakabeppu, T. Morisaki and K. \\ Yoshioka
}

We previously reported that c-Jun $\mathrm{NH}_{2}$-terminal kinase (JNK)/stress-activated protein kinase-associated protein 1 (JSAP1), a scaffold protein for JNK signaling pathways, is important in embryonic stem (ES) cells during neurogenesis. In that study, we also observed altered expression of mesodermal marker genes, implying that JSAP1 is involved in differentiation during mesodermal lineages. In the present study, we investigated the function of JSAP1 focusing on cardiomyocyte development using JSAP1-null ES cells, and found that cardiomyogenesis is impaired in the JSAP1-null mutant. We also found that JSAP1-deficiency results in lower expression of the cardiac transcription factor $\mathrm{Nkx} 2.5$ and contractile proteins, and contrarily, in significantly higher expression of mesoderm-related markers other than the cardiomyocyte lineage. Taken together, these results suggest that JSAP1 may play an important role in differentiation during mesodermal lineages, where JSAP1 functions as a positive factor for cardiomyocyte differentiation, and as an inhibitory factor for differentiation into the other lineages.

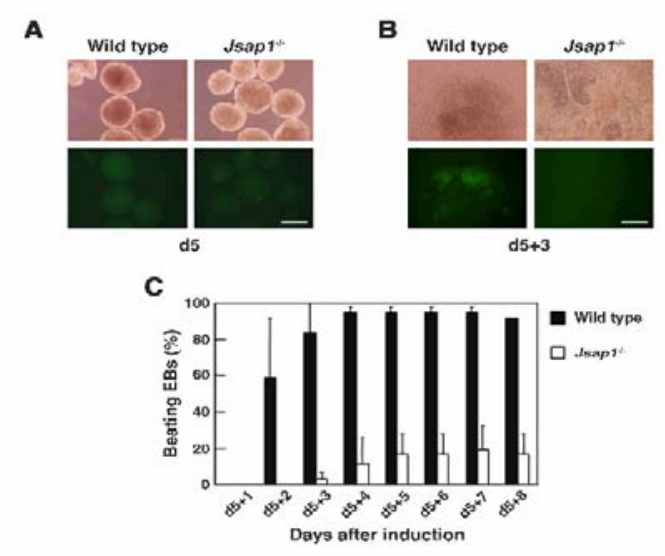

Figure Impairment of cardiomyogenesis in $J_{s a p} 1^{-/-}$Nkx2.5GFP ES cells. (A) EBs derived from wild-type and $J_{S a p} 1^{-/-}$ES cells were almost identical morphologically at d5. Phase-contrast photomicrographs of the EBs (upper panels) and the corresponding fluorescent micrographs (lower panels) are shown. Scale bar represents $250 \mu \mathrm{m}$. (B) Cardiomyogenesis of $J_{\text {sapl }} 1^{-/-}$ES cells was largely impaired at d5+3. Micrographs are as in (A). Scale bar represents $250 \mu \mathrm{m}$. (C) Quantification of contracting EBs derived from wild-type and $J_{S a p 1^{-/-}}$ES cells.

Reference: T. Sato, K. Hidaka, A. Iwanaga, M. Ito, M. Asano, Y. Nakabeppu, T. Morisaki and K. Yoshioka (2005) BBRC, 338, 1152-1157. 